\title{
Staphylococcal Septicemia, Purulent Pericardial Effusion Causing LV Pseudo-aneurysm in Children: A Case Report

\author{
Mohd Iqbal Dar ${ }^{1 *}$, Aamir Rashid ${ }^{2}$, Zubair Mushtaq Tramboo ${ }^{3}$, Nagraju Naik Banavath ${ }^{1}$, Imran Hafeez ${ }^{2}$, Hilal A Rather ${ }^{2}$
}

${ }^{1}$ senior Resident, Department of Cardiology, SKIMS soura Srinagar, J\&K, India

${ }^{2}$ Consultant Cardiology Department of Cardiology, SKIMS Soura, J\&K, India

${ }^{3}$ Senior Resident, Department of Paediatrics, SKIMS soura Srinagar, J\&K, India

DOI: $10.36347 /$ simcr.2020.v08i11.007

| Received: 25.10.2020 | Accepted: 07.11.2020 | Published: 11.11.2020

*Corresponding author: Mohd Iqbal Dar

\section{Abstract}

Cardiac pseudoaneurysm formation following isolated pericardial effusion is one of the rare complications with only few case reports published so far in the literature. A $3 \frac{1}{2}$ years old male child present with fever and purulent polyarthritis causing staphylococcus aureus septicemia, which finally complicated into purulent pericardial effusion followed by left ventricular pseudo-aneurysm formation. Despite aggressive management, the patient had a downhill course of illness and finally resulted in death. Staphylococcal infection, especially in children can lead to metastatic infection of heart with fatal outcomes.

Keywords: LV pseudo-aneurysm, pericardial effusion, staphylococcal septicaemia, case report.

Copyright $(\mathcal{2 0 2 0}$ The Author(s): This is an open-access article distributed under the terms of the Creative Commons Attribution 4.0 International License (CC BY-NC 4.0) which permits unrestricted use, distribution, and reproduction in any medium for non-commercial use provided the original author and source are credited.

\section{INTRODUCTION}

Development of cardiac pseudoaneurysm is usually restricted to post-cardiac surgery scenario or complicated coronary artery disease and is associated with significant morbidity and mortality. The data on the development of LV pseudo-aneurysm secondary to staphylococcal infection in children is scanty and this case report is intended to highlight the potential adverse outcomes of this condition.

\section{Case Presentation}

A 3 1/2 years old male child presented with 10 days history of fever and painful swollen joints to a peripheral hospital. Aspiration of joint showed pus and the patient was put on broad-spectrum antibiotics. His fever persisted and started to have breathlessness on effort and hemodynamic instability. Initial investigations showed cardiomegaly on chest x-ray. Echocardiography done showed a large pericardial effusion with thick septation's suggestive of Pyopericardium and a mild mitral regurgitation with no valvular vegetation's. Patient was shifted on day 14 of the illness for further management to our institute. Echocardiography at our centre done was suggestive of large pericardial effusion with septation's and no evidence of any valvular vegetation's as shown in Figure-1.

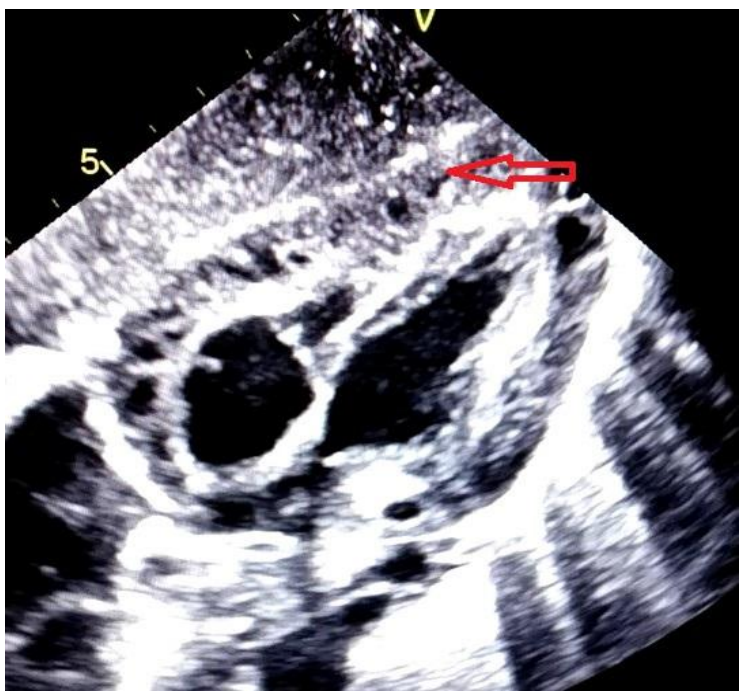

Fig-1: Echocardiography showing large pericardial effusion with thick echogenic effusiate suggestive of Pyopericardium (Arrow)

Patient underwent partial pericardectomy on the next day and a pericardial window was made. Intraprocedural findings were suggestive of thickened, inflamed and adherent pericardium to the underlying myocardium. Adhesiolysis and pus drainage was done. Blood cultures and pus cultures were suggestive of MRSA with sensitivity to vancomycin, linezolid, teicoplanin and clindamycin. Antibiotic treatment was modified as per sensitivity. Patient had initial 
hemodynamic improvement but patient persisted to be febrile. A repeat echo done on day 24 of illness showed large pericardial effusion with thick septation's, severe mitral regurgitation and no features of tamponade, no vegetations. Next day patient developed acute respiratory distress and heart failure. After reassessing, the patient was taken for complete pericardectomy. The intra-operative findings showed thickened and adherent pericardium to the right atrium and right ventricle, minimal pus and aneurysmal dilatation of left ventricle. Post-surgery Echocardiography revealed a 26x23mm submitral aneurysm (Figure-2) on the lateral aspect of the left ventricle with free flow of blood into \& out of it, severe MR (Figure -3) and minimal organized pericardial effusion.

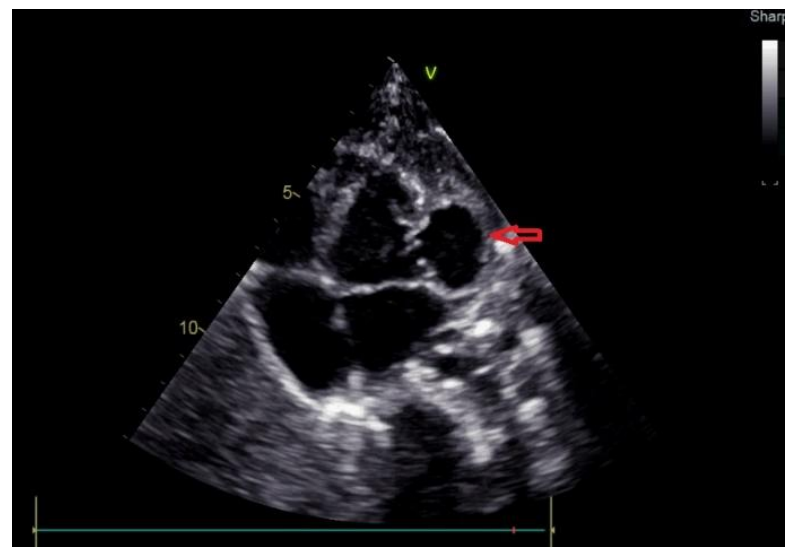

Fig-2: Echocardiographic apical four-chamber view showing LV Pseudo-aneurysm (Arrow)

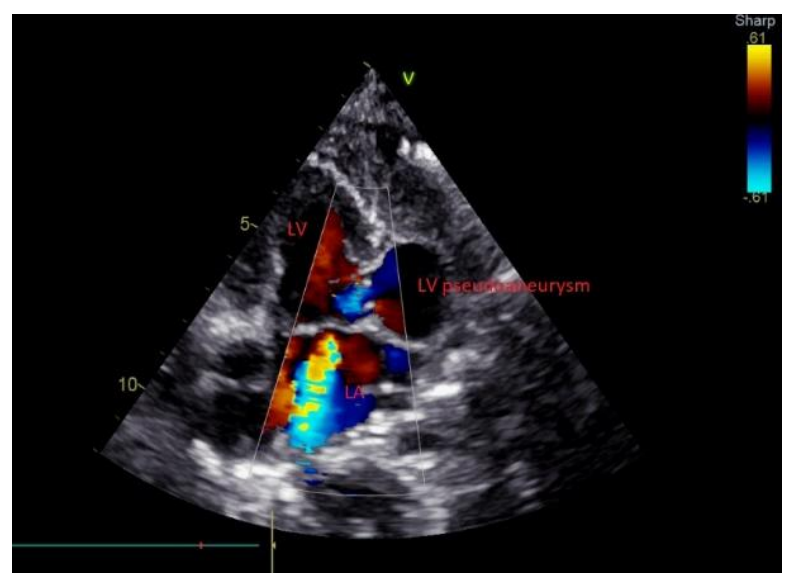

Fig-3: Colour Doppler showing pseudoaneurysm to LV communication and sever MR

From $3^{\text {rd }}$ postoperative day, condition of the patient deteriorated further with worsening heart failure and hemodynamics. Despite supportive management and inotropic support, his conditions did not improve and the patient succumbed to the illness.

\section{DISCUSSION}

Cardiac pseudoaneurysm by definition is ruptured myocardium which is contained by pericardium or adhesions and allowing to and fro flow of blood from ventricular cavity [1]. These are found incidentally on follow-up or represent part of complication during the process of the disease. A systematic review done by mayo clinic [2] on the natural history of cardiac pseudoaneurysm revealed that most of these pseudoaneurysms are found incidentally on follow-up, with acute myocardial infarction and post-cardiac surgery being two main aetiologies. The location of these pseudoaneurysms can represent the likely aetiology like infero/postero lateral LV wall following an infraction, RVOT following complex congenital surgeries, posterior subannular region of the mitral valve and subaortic area following MVR and AVR surgeries and mitral-aortic intervalvular fibrosa following IE. Other than these pseudoaneurysms can occur as a part of disease processes like trauma, syphilis, rheumatic carditis and disseminated tuberculosis. Clinical presentation could vary from asymptomatic incidental finding to recurrent acute chest pain, acute heart failure, arrhythmias, syncope and systemic embolization. The outcome of these patients solely depends on the nature of the underlying disease, prompt diagnosis and timely intervention.

The literature on paediatric age group presenting as isolated pericarditis complicated with pseudoaneurysm is scarce with only a few handful of cases reports so far [3-8]. All the cases reported are associated with Staphylococcus aureus Septicemia, presenting initially as purulent pericardial effusion and complicated with LV aneurysm formation over 2-4 weeks of disease course. Possible mechanisms for pseudoaneurysm formation proposed were direct myocardial inflammation from the pericardium, myocardial abscess formation as part of septicaemia, endocarditis and abscess formation and possible staphylokinase activity with collagen degradation [8]. All leading to myocardial disruption leading to rupture and pseudo aneurysm formation. No definitive association between particular mechanisms could be demonstrated so far, except all these patients reported have Staphylococcus aureus Septicemia and a pseudoaneurysm formation by $2-4$ weeks. Few case reports have also suggested that this myocardial abscess formation is responsible for cardiac rupture and Sudden Cardiac Death [9]. Most of the patients with pseudoaneurysm suffer grave prognosis unless intervened timely with the closure of pseudoaneurysm.

In our case, we assume that the initial purulent arthritis was likely caused by Staphylococcus aureus which after arthrotomy led to septicaemia. Both the preoperative blood and post-operative pus cultures support Staphylococcus aureus septicemia. Persistence of fever, not much clinical improvement despite pericardial drainage by partial pericardectomy and deterioration of the condition with severe MR and no endocardial involvement favours myopericarditis and possible multiple micro abscess formation which later led to a rupture and pseudoaneurysm formation. 


\section{CONCLUSION}

Staphylococcal septicemia in children can lead to cardiac infection and complicate into LV pseudoaneurysm formation. The anticipation of this condition in these circumstances can help in better management of these patients.

\section{Abbreviations}

LV- Left Ventricle, MRSA- MethicillinResistant Staphylococcus Aureus, RVOT- Right Ventricular Outlet, MVR-Mitral Valve replacement, AVR- Aortic Valve Replacement, IE- Infective endocarditis

\section{REFERENCES}

1. Hurst CO, Fine G, Keyes JW. Pseudoaneurysm of the heart. Report of a case and review of literature. Circulation. 1963; 28:427-36.

2. Yeo TC, Malouf JF, Oh JK, Seward JB. Clinical Profile and Outcome in 52 Patients with Cardiac Pseudoaneurysm. Ann Intern Med.1988; 128:299305.

3. Moraes AN, Ferreira AG Jr, Gomes Ferreira SMA. Left ventricular pseudoaneurysm complicating infective pericarditis. Heart. 1999;82: 393-394
4. Boer HD, Elzenga NJ, Boer WJ, Meuzeleer JJ. Pseudo aneurysm of the left ventricle after isolated pericarditis and Staphylococcus aureus septicaemia. Eur J Cardiovasc Surg. 1999; 15: $97-$ 99.

5. Gaur L, Madan S, Morell V, Arora G. Formation of ventricular aneurysm after endocarditis in a child with methicillin resistant Staphylococcus aureus bacteraemia. Pediatr Cardiol. 2011; 32:1062-1064.

6. Juneja R, Sharma R, Kothari SS. Sub mitral pseudo aneurysm in purulent pericarditis. Heart. 2000; 83: 713.

7. Vinitha VN, Dhananjay M, Kothari SS. Unusual left ventricular pseudoaneurysm in a child after disseminated bacterial infection. World $\mathrm{J}$ Pediatr Cardiac Surg. 2014; 5: 121-123.

8. Desai M, Gandhi H, Mishra A. Post-infective pericarditis left ventricular pseudoaneurysm: a case report and review of literature. Cardiology in the young. 2015; 25,358-361.

9. Abela GS, Majmudar B, Felner JM. Myocardial abscesses unassociated with infective endocarditis. South Med J. 1981; 74:432-4. 